Article

\title{
An Examination of the Associations Among Multiple Memory Systems, Past Tense, and Vocabulary in Typically Developing 5-Year-Old Children
}

\author{
Jarrad A. G. Lum ${ }^{a}$ and Evan Kidd ${ }^{b}$
}

\begin{abstract}
Purpose: Considerable research has investigated the role of verbal working memory in language development in children with and without language problems. Much less is currently known about the relationship between language and the declarative and procedural memory systems. This study examined whether these 2 memory systems were related to typically developing children's past tense and lexical knowledge.
\end{abstract}

Method: Fifty-eight typically developing children approximately 5 years of age completed a battery of linguistic and nonlinguistic tasks, including tests of vocabulary, past tense production, and procedural and declarative memory.
Results: The results showed that declarative and procedural memory were not correlated with either regular or irregular past tense use. A significant correlation was observed between declarative memory and vocabulary.

Conclusions: The results of the study were not consistent with the view that the declarative and procedural memory systems support children's use of the regular and irregular past tense. However, evidence was found suggesting that declarative memory supports vocabulary in this age group.

Key Words: past tense, vocabulary, declarative memory, procedural memory
C hildren's use of the past tense has been studied to address a range of issues in language development. For example, children's production of past tense forms has been used to examine whether children's linguistic behavior is rule driven or schema based (Bybee \& Slobin, 1982; Pinker, 1991). Other research has focused on whether the acquisition of the past tense proceeds independently or is dependent on advances in other linguistic domains (Bates \& Goodman, 1997; van der Lely \& Ullman, 2001) and domain-general cognitive abilities (Joanisse \& Seidenberg, 1998). The study of past tense has also been used to examine the relationship between

\footnotetext{
${ }^{\mathrm{a}}$ Deakin University Australia, Melbourne, Victoria

${ }^{\mathrm{b}}$ The Australian National University, Canberra, Australia

Correspondence to Jarrad A. G. Lum: jarrad.lum@deakin.edu.au

Editor: Janna Oetting

Associate Editor: Sean Redmond
}

Received May 24, 2010

Revision received October 28, 2010

Accepted November 4, 2011

DOI: $10.1044 / 1092-4388(2011 / 10-0137)$ language and memory (Bishop, Adams, \& Norbury, 2006; Pinker \& Ullman, 2002). One proposal forwarded by Ullman (2001a, 2001c, 2004) is that procedural memory supports the acquisition and use of the regular past tense (and grammar in general) and that the declarative memory system supports irregular verbs and nouns as well as lexical items. To date, little research has been undertaken to examine associations between language and the declarative and procedural memory systems in typically developing children. This gap was addressed in the present study by examining the relationship between declarative and procedural memory, past tense, and vocabulary in typically developing 5 -year-old children.

\section{The Declarative and Procedural Model of the Lexicon and Grammar}

The declarative and procedural model of the lexicon and grammar (hereafter referred to as the $D \& P$ model) proposed by Ullman (2001a, 2001c, 2004) attempts to account for language acquisition and processing in both typical and atypical populations (e.g., Hartshorne 
\& Ullman, 2006; Ullman, 2001b; Ullman et al., 2005; Ullman \& Pierpont, 2005; Walenski, Motofsky, \& Ullman, 2007; Walenski, Sosta, Cappa, \& Ullman, 2009; Walenski, Weickert, Maloof, \& Ullman, 2010). A summary of the memory systems implicated by the D \& P model and their hypothesized role in language is presented in Table 1.

Declarative and procedural memory are long-term memory systems; both can store information on timescales that can last from minutes to years. This can be contrasted with working memory, which holds information on the order of seconds (Baddeley, 2003; Cowan, 1997; Engle, Cantor, \& Carullo, 1992). Distinct neurological structures supporting declarative and procedural systems have been identified along with functional differences in learning and memory processes. The declarative system is principally supported by the medial temporal lobes (Mayes, Montaldi, \& Migo, 2007; Squire, Stark, \& Clark, 2004). Encoding and retrieval via this system largely proceed via association, in which two or more arbitrarily related elements can be used to form a single memory trace. This system is typically implicated in the storage of semantic information (e.g., knowledge of objects and events) and episodic events (e.g., personal experiences). Learning via the declarative memory system is fast and can be achieved after a single exposure. However, the strength of stored representations is increased after multiple exposures, which in turn facilitates retrieval of information from this system.

Procedural memory is primarily supported by a network that principally includes basal ganglia, cerebellum, and prefrontal cortex (Hikosaka, Nakamura, Sakai, \& Nakahara, 2002; Packard \& Knowlton, 2002; Robertson, Tormos, Maeda, \& Pascual-Leone, 2001). Initially, this memory system was considered to support the acquisition of motor skills and habits but was later demonstrated to be involved in higher order operations (e.g., Knowlton, Mangels, \& Squire, 1996). Information acquired or retrieved via the procedural system can be accessed without conscious reflection. Unlike the declarative memory system, learning occurs through repeated exposures rather than from a single exposure. For example, the acquisition of new motor skills requires repetition, and learning the association between two probabilistically related events can be achieved only after multiple presentations. Once information has been acquired via the procedural memory system, skills can be executed and knowledge demonstrated without awareness.

In broad terms, the D \& $\mathrm{P}$ model holds that declarative memory is the repository of lexical facts, including word meanings and other arbitrary linguistic information not predictable from mental rules, such as irregular verb formation (e.g., go $\rightarrow$ went, sing $\rightarrow$ sang) and highfrequency frozen or semiproductive constructions (e.g., Ullman, 2001c, 2004). This suggestion derives from the fact that the declarative memory system is well suited to binding or associating arbitrarily related information. Procedural memory is proposed to be responsible for those aspects of language that involve rule-based computations, such as the formation of the past tense of regular verbs (walk $\rightarrow$ walked) or the formation of the passive construction (e.g., the dog was walked by the man). This memory system is argued to be principally involved in the learning of grammatical rules because it is better suited to detecting, storing, and retrieving information that is statistically, probabilistically, or sequentially structured.

\section{Interactions Between Memory Systems and Past Tense Use}

Evidence has been presented demonstrating that declarative and procedural memory interact during learning and memory (e.g., Foerde, Knowlton, Poldrack, $\&$ Smith, 2006; Poldrack et al., 2001; for reviews, see Poldrack \& Packard, 2003; Poldrack \& Rodriguez, 2004). Specifically, depending on the material to be learned or retrieved, these two memory systems may cooperate or compete. In cases of cooperation, it has been observed

Table 1. Summary of the memory systems and proposed role in language implicated in the declarative and procedural (D\&P) model.

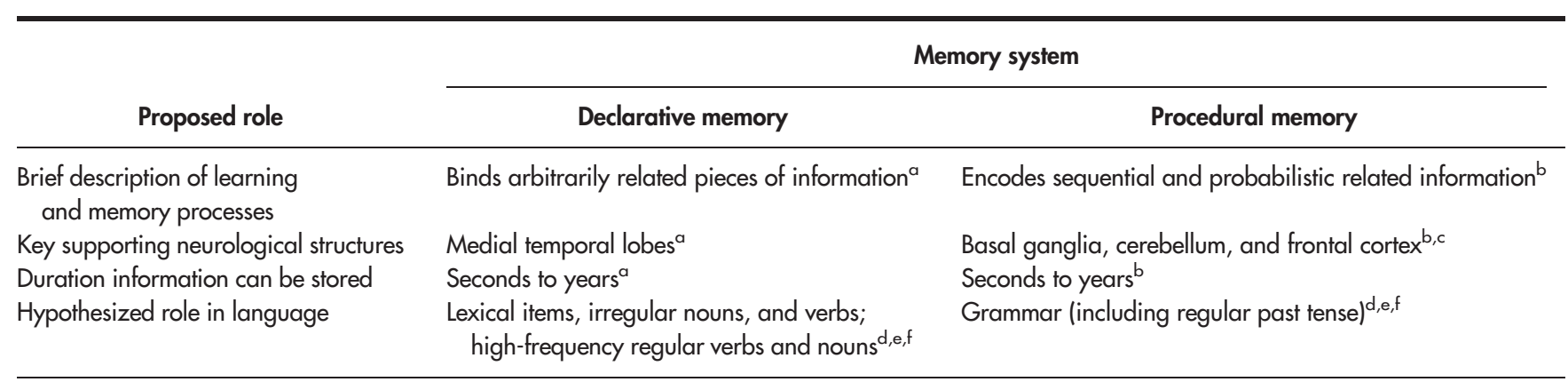

aSquire, Stark, and Clark (2004). ' Packard and Knowlton (2002). 'Robertson, Tormos, Maeda, and Pascual-Leone (2001). dUllman (2001a, 2001b, 2001c). eUllman (2004). 'Hartshorne and Ullman (2006). 
that both declarative and procedural memory systems are active during learning. Furthermore, in instances of cooperation, it has been observed that the declarative system is involved in the early stages of learning, with procedural memory becoming involved after repeated training (e.g., Iaria, Petrides, Dagher, Pike, \& Bohbot, 2003). Competition between memory systems has been characterized as activation in one memory system that is simultaneously accompanied by deactivation in the other (e.g., Poldrack \& Gabrieli, 2001). In instances of competition, evidence has been presented suggesting that if working memory is disengaged from the learning process, information is likely to be memorized by the procedural memory system (Foerde et al., 2006). Foerde et al. showed that at a functional level - and assuming no problems with the basal ganglia-declarative memory may be more dependent on working memory than procedural memory.

The D \& P model takes into account interactions between memory systems (Ullman, 2001c, 2004). The interactions between declarative and procedural systems rule out the possibility that the production and acquisition of all regular past tense forms represent the functions of a single system. Thus, within the D \& $\mathrm{P}$ model, the learning and use of past tense can be viewed as the product of cooperative and competing processes between memory systems. In relation to cooperative interactions, Ullman (2001c, 2004) speculated that early in the language acquisition process, children may acquire some items declaratively before the information is transferred to the procedural memory system. Thus, depending on the age of the child, declarative or procedural memory may support past tense use.

According to the D \& P model (Ullman, 2001a, 2001b, 2001c, 2004), competition between declarative and procedural memory systems occurs during past tense use. For instance, in the production of regular or irregular past tense, the declarative memory system attempts to find the target verb among stored lexical items, and the procedural memory system attempts to apply a rule. If the procedural system is able to apply a rule to create the past tense form, this inhibits the functions of the declarative memory system. The outcome of this process is the affixation of the regular past tense inflection to a verb stem. However, if the targeted verb is found in declarative memory, the processes of the procedural memory system will be overridden, and the application of the rule will be "blocked" (see Marcus et al., 1992). In some cases, competition between systems will produce errors. If the speaker's goal is to produce an irregular verb, but this item cannot be found in declarative memory, the procedural memory system will apply a rule leading to an over-regularization error.

According to the $\mathrm{D} \& \mathrm{P}$ model, the frequency and phonological form of the verb may also play a role in determining the involvement of the declarative or procedural memory system in past tense use. As noted earlier, repetition increases the strength of information stored in the declarative memory system. As such, the D \& $\mathrm{P}$ model predicts that high-frequency regular verbs may be stored in declarative memory (Ullman, 2001a, 2001c). Thus, the frequency of an item is an important factor influencing whether it is stored and retrieved procedurally or declaratively. Ullman and colleagues (Hartshorne \& Ullman, 2006; Ullman, 2001a, 2001c) have also suggested that the declarative memory system may produce over-regularization errors for irregular verbs that are phonologically similar to regular verbs. Specifically, it is claimed that in cases where the declarative memory system has stored a large number of regular verbs, the regular past tense suffix may be applied to irregular items that have also been stored in this memory system. This may occur via an associative network whereby activation of an irregular past tense verb also activates similar sounding regular past tense forms (e.g., hold-holded, cf. fold-folded, mold-molded; examples from Hartshorne \& Ullman, 2006, p. 25).

\section{Language Development and the Declarative and Procedural Memory Systems in Typically Developing Children}

To date, there have been few empirical tests of the D \& P model undertaken with nonclinical pediatric populations. Rather, key research supporting this position has been presented from studies of adults with neurodegenerative disorders or children with developmental language disorders. For instance, adults with neural damage affecting the basal ganglia and subsequently procedural memory have been observed to perform more poorly than controls on tasks requiring the use of regular past tense (Ullman et al., 1997, 2005). In contrast, adults with damage to the temporal lobes have been reported to perform more poorly on the use of irregular past tense and measures of lexical knowledge (Walenski et al., 2009). In specific language impairment (SLI), Tomblin, Mainela-Arnold, and Zhang (2007) found that a measure of procedural memory correlated with grammatical but not lexical knowledge in a sample comprising adolescents with and without SLI. Whether the associations between memory and language predicted by the D \& P Model can be observed in nonclinical pediatric populations is yet to be thoroughly tested.

When considering the role of declarative and procedural memory in language, the development of these systems might also need to be taken into account. Reber (1993) proposed that the procedural memory system may reach functional maturity earlier than declarative memory. There is both neurological and behavioral evidence 
to support this claim. Neurological evidence indicates that the processes of cell migration, synaptogenesis, and myelination are completed earlier in subcortical structures, including those supporting procedural memory, than in frontal and temporal structures implicated in declarative memory (Anderson, Elisabeth, Hendy, \& Wrennall, 2001; Giedd et al., 1999). In behavioral studies, age-related improvements have been observed for tasks measuring declarative memory (Anderson \& Lajoie, 1996; Kramer, Delis, Kaplan, O’Donnell, \& Prifitera, 1997; Lum, Kidd, Davis, \& Conti-Ramsden, 2010) but not always for procedural memory (Lum, Kidd, et al., 2010; Meulemans, Van der Linden, \& Perruchet, 1998; Thomas \& Nelson, 2001; for alternative findings, see Thomas et al., 2004). The impact of brain development on memory interactions is poorly understood, as research reported to date involves adult human and nonhuman animals (for a review of the adult literature, see Poldrack \& Rodriguez, 2004). From the perspective of the D \& P Model, it could be predicted that because procedural memory develops first, this system may have a more influential role in language development, especially in relation to the acquisition and use of regular past tense forms. Subsequently, from an empirical perspective, finding an association between procedural memory and regular past tense use might be more likely to be observed in young, typically developing children.

\section{The Present Study}

The aim of this study was to examine whether measures of declarative and procedural memory would be correlated with measures of past tense and vocabulary in typically developing 5 -year-old children. In the context of developmental language disorders/impairments such as SLI, understanding the mechanisms underlying past tense is particularly important given that deficits in this aspect of language appear to be a clinical marker for this population (e.g., Rice, 2000). Subsequently, attempts to specify the underlying mechanisms of past tense in typical development may also provide insights into the general nature of the language problem in atypical populations as well.

In this study, children were presented with a battery of memory tests that assessed declarative and procedural memory. In addition, children were presented with tasks assessing past tense and vocabulary. Children's production of past tense was measured using a task developed by Marchman, Wulfeck, and Weismer (1999). This task assesses production of both regular and irregular verbs. The items are coded for frequency and neighborhood density. Specifically, irregular verbs are classified as having either a high or low number of "regular enemies." Irregular verbs identified as having a high number of regular enemies are phonetically similar to a larger number of regular verbs. Using this task, Marchman et al. reported that in typically developing children, over-regularization errors were more likely to occur for low-frequency items and those with a high number of regular enemies. It was also found that zeromarking of irregular and regular verbs was more likely to occur for low-frequency items. Zero-marking describes instances where children produce the infinitive form of the verb in contexts that require the past tense form. Marchman et al.'s past tense task can be considered useful in investigating the claims of the D \& $\mathrm{P}$ model. This is because the performance of children on this task can be explained with reference to the D \& P model.

The children participating in the study were approximately 5 years old. This age group was selected for two reasons. First, in this study, children were presented with neuropsychological tasks that assessed declarative and procedural memory. These tasks, especially the task assessing procedural memory, required children to maintain attention for relatively long periods of time. (A description of the task used to examine procedural memory is presented in the Method section.) Second, research has shown that children's production of irregular verbs does not reach ceiling at this age (Rice, Wexler, Marquis, \& Hershberger, 2000). In relation to regular past tense production, past research has shown that in typically developing children, the average score approaches ceiling on regular past tense elicitation tasks (Rice \& Wexler, 1998). However, research also suggests that variance at this age is sufficiently large to expect that not all typically developing children have acquired regular past tense forms. For example, Rice, Wexler, and Cleave (1995) and Rice and Wexler (1996) observed that the mean proportion of correct responses to a past tense elicitation task for 5-year-olds was around $92 \%$ in both studies. However, the $S D$ in the two studies was found to be $14 \%$ and $18 \%$, respectively. Interestingly, longitudinal research has shown that the variance in past tense use decreases considerably after 5 years of age. Rice and Wexler (1998) observed the $S D$ to be $8 \%$ in 6 -year-olds and $2 \%$ in 7-year-olds. Thus, it seems that in typical development, 5-year-olds may be the oldest age group in which errors in regular past tense may appear but are not necessarily indicative of a language impairment or delay.

\section{Hypotheses}

The hypotheses forwarded in this study were based on the proposal by Ullman (2001a, 2001c, 2004) that individual differences in declarative memory functioning may be associated with differences in language functioning (e.g., Hartshorne \& Ullman, 2006). Following a similar logic, individual differences in procedural memory functioning should have an observable influence in 
relation to the suffixation of regular verbs. That is, those children with relatively better procedural memory functioning should seize upon the regular pattern more quickly. Based on this past work, the following hypotheses were forwarded. Declarative memory was predicted to be positively correlated with (a) vocabulary size and (b) correct production of irregular verbs. Procedural memory was predicted to be correlated with the production of the regular past tense, especially low-frequency items. The D \& $\mathrm{P}$ model also makes the following predictions based on the frequency and phonology of the verb. Declarative memory was predicted to be (a) correlated with highly frequent regular verbs and (b) positively correlated with overregularization errors for irregular verbs that have a high number of regular enemies.

\section{$\overline{\text { Method }}$ \\ Participants}

Fifty-eight monolingual English-speaking children (30 female, 28 male) participated in the study. The mean age of the sample was 65.38 months $(S D=3.8$, range $=59-73$ ). The children were recruited from elementary schools within Greater Manchester, United Kingdom. None of the children were receiving support for a language or learning-related problem.

\section{Materials}

In this study, children were presented with tasks measuring declarative memory, procedural memory, past tense, and vocabulary. Children were also presented with the Raven's Colored Progressive Matrices (RCPM; Raven, 1998) and Children's Test of Nonword Repetition (CNRep; Gathercole \& Baddelely, 1996) to provide evidence that children were not language impaired or developmentally delayed. Each test is now described in turn.

Measure of test of nonverbal intelligence. The RCPM (Raven, 1998) test was used to provide a nonverbal assessment of children's general intellectual functioning. This test has positive correlations with the Performance and Verbal IQ scales $(r \approx .5)$ from the Wechsler Intelligence Scale for Children (Chalmers \& Frederick, 1955). Scores from this test are standardized to a mean of 100 and an $S D$ of 15 . All children participating in the study obtained a standard score of 85 or greater. In addition, scores from the RCPM were used as a covariate in the correlation analyses examining associations between the language and memory measures.

Measure of nonword repetition. Children were presented with the CNRep (Gathercole \& Baddelely, 1996). Nonword repetition tasks have been proposed to index the short-term storage functions of working memory. It is acknowledged that whether nonword repetition can be considered to assess one component of working memory or the entire system depends, in part, on the model of working memory adopted. For instance, in Baddeley and Hitch's model of working memory (Baddeley, 2003), there are separate components termed the visuospatial sketchpad and phonological loop that are responsible for the short-term storage of visual and verbal information, respectively. It has been proposed that nonword repetition tasks provide a measure of the phonological loop component (Gathercole, 1995; Gathercole, Pickering, Ambridge, \& Wearing, 2004). Alternatively, in models that propose the short-term storage and processing functions are supported by a single component (e.g., Engle et al., 1992), nonword repetition tasks can be considered to index the entire system. Thus, whereas the nonword repetition task requires the short-term storage of verbal information, it may also measure other components of working memory as well.

The CNRep was used to screen for language impairments in the sample. Considerable research has been presented indicating that children with language problems have particular difficulty with nonword repetition tasks (Bishop, North, \& Donlan, 1996; Conti-Ramsden, Botting, \& Faragher, 2001; Weismer et al., 2000; for a recent meta-analysis, see Graf Estes, Evans, \& Else-Quest, 2007). In the CNRep, children are asked to repeat 40 nonwords that vary in length from two to five syllables. Each syllable length is assessed with 10 nonwords. In this study, the nonwords were prerecorded and presented to children over headphones. In the CNRep, words are presented one at a time and, after the child has responded, the next nonword is presented. Children's responses were audiotaped and coded for accuracy offline. A response was counted as correct if the child correctly reproduced the entire nonword. The test has been standardized to a mean of 100 and an $S D$ of 15 . All children participating in this study obtained a standard score of 85 or greater.

Measure of declarative memory for verbal information. The Word Pairs subtest from the Children's Memory Scales (CMS; Cohen, 1997) was used to measure declarative memory. This task is appropriate for children as young as 5 years (Cohen, 1997). Performance on this task has been shown to be related to the left medial temporal lobe functioning, and the task is considered appropriate to measure declarative memory (for neuropsychological evidence for the word pairs task used in this study, see Cohen, 1997; e.g., Eichenbaum \& Bunsey, 1995; Jambaqué et al., 2007; Wood et al., 2000).

In this task, children are asked to learn a single list consisting of 10 semantically unrelated word pairs (e.g., nurse-fire). Children are given three exposure trials. At 
the start of each trial, the list of word pairs is presented orally. After each list has been presented, children are provided with the first word of the pair (e.g., nurse) and asked to recall the second (e.g., fire). This procedure is followed for the second and third presentations of the list. At the conclusion of the third trial, there is another recall task in which participants are asked to recall both words in the pair. Children's performance on this task was measured by summing the total number of correct responses over all trials as outlined in the CMS test manual. The highest raw score obtainable on this task is 40 .

Measure of procedural memory. Procedural memory was tested using a version of Nissen and Bullemer's (1987) Serial Reaction Time (SRT) task. In this task, participants implicitly learn a repeating sequence. For readers unfamiliar with SRT tasks, a brief summary of the task and hypothetical results are presented before describing the details of the SRT task used in the present study.

In SRT tasks, participants implicitly learn a visuospatial sequence. Specifically, a visual stimulus repeatedly appears in one of four marked locations on a computer display, and participants are asked to press one of four buttons on a response pad that correspond to the location of a visual stimulus (e.g., Nissen \& Bullemer, 1987). The main dependent variable of interest in SRT tasks is a participant's reaction time (RT), which is the amount of time taken to press a response button after presentation of the visual stimulus. The only instruction provided is to press the response button that matches the location of the visual stimulus. Visual stimulus presentations are usually divided into blocks, with each block comprising approximately $60-100$ visual stimulus presentations. Unknown to participants, in several blocks the visual stimulus follows a sequence, but in others, the visual stimulus appears randomly. Hypothetical results for neurologically intact participants are presented in Figure 1.

In nonclinical populations, button presses become faster (i.e., RTs decrease) as more trials containing the repeating sequence are presented. However, when the visual stimulus appears randomly, button presses become slower (i.e., RTs increase; (e.g., Thomas et al., 2004). The increase in RTs is taken as evidence that knowledge about the sequence has been acquired. If no information had been acquired, we would expect RTs to decrease or reach asymptote. Individuals with neurodegenerative conditions affecting the basal ganglia typically do not show an increase in RTs that is of the same magnitude as that of controls when the visual stimulus appears randomly (e.g., Knopman \& Nissen, 1991; Nissen \& Bullemer, 1987; for a meta-analysis examining SRT task performance in Parkinson's disease, see Siegert, Taylor, Weatherall, \& Abernethy, 2006). However, individuals with brain damage affecting other parts of the brain do show an increase in RT comparable to that of controls (Nissen \& Bullemer, 1987).

In the present study, children completed a version of the SRT task using a Gravis Gamepad Pro as a response pad, which was connected to a Dell Latitude C500 laptop computer. The response pad consisted of four buttons arranged in the shape of a diamond, which children operated using their right thumb. The decision to use this response format was motivated by the need to maintain children's interest in the task. It should be noted that having children use the response pad was not considered

Figure 1. Hypothetical results for neurologically intact participants.

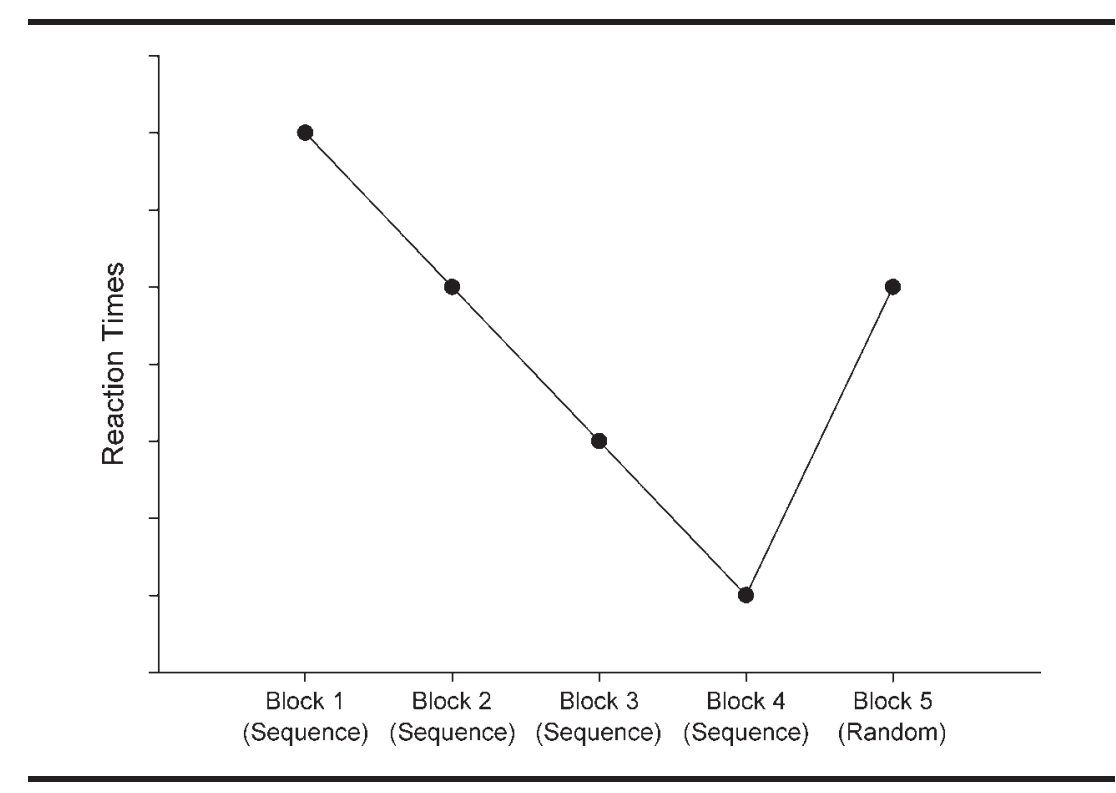


to be a problem for left-handed children. It has been found that handedness is not related to performance on the task (Lum, Gelgec, \& Conti-Ramsden, 2010).

Tests of procedural memory require a large number of trials. The response pad also enabled us to present the SRT task to the children in a context of a computer game, which served to pique the children's interest in the task. The visual stimulus consisted of a well-known cartoon character, which appeared in one of four spatial locations presented on the computer monitor with a black background. Four boxes marked the spatial locations on the monitor with white boarders. These boxes were arranged in a manner identical to the location of the buttons on the response pad (i.e., a diamond configuration). During testing, children sat approximately $40 \mathrm{~cm}$ away from the computer screen. The white boxes subtended a visual angle of $6.4^{\circ} \times 6.4^{\circ}$.

At the start of the task, children were told that the cartoon character would appear in one of four places on the computer display, and their task was to press the button that matched the character's location. Ten practice trials were then presented to ensure the children understood the task. All children obtained an accuracy level of at least $90 \%$ on the practice trials. After this, the test trials were then presented, which comprised five blocks of 60 trials.

Unbeknownst to the children, on Blocks 1-4, the appearance of the visual stimulus followed a 10-item sequence. Labeling the spatial locations 1 through 4 in a clockwise manner, starting with the leftmost position of the diamond as 1 , resulted in a repeating sequence of $4,2,3,1,3,2,4,3,2,1$. This sequence was the same used by Nissen and Bullemer (1987). The presentation of the stimulus in the fifth block was presented in a pseudorandom order, with the following two constraints. First, in this block the visual stimulus appeared in each spatial location an equal number of times as for each of the preceding blocks. Second, the probability of observing pairs of items within the repeating sequence was the same. These probabilities were also maintained in the random block. Introducing this constraint meant we were able to control for the possibility that differences in performance between the repeating and pseudorandom blocks arose form learned associations between pairs of stimulus transitions.

Accuracy and RTs from this task were used in the data analysis. Accuracy was computed as the proportion of times children pressed the correct button after presentation of the visual stimulus. These data were obtained to examine whether children were responding appropriately to the visual stimulus. Because RTs were used to index children's learning on the task, it was also necessary to control for individual differences in motor response speeds. This was achieved by normalizing individual
RTs using a method outlined by Thomas et al. (2004). In this approach, each child's RTs across all blocks on the SRT task were converted to a $z$ score that was referenced to the median and $S D$ of all their RTs from the task. The average $z$ score for each block was then computed. Thus, each child's performance on the SRT task was described with separate normalized RTs computed for Blocks 1-5.

After completing the SRT task, the children were presented with a recall task that assessed their awareness of the pattern. In past research using SRT tasks (e.g., Nissen \& Bullemer, 1987), adults have typically been asked whether they detected a pattern during the test trials. Those participants who respond in the affirmative were asked to generate the sequence. Adults who are able to consciously recall the pattern are then excluded from the data analysis. Adhering strictly to this protocol with children was considered problematic given that they may be inclined to provide a prosocial response. In our recall task, children were not asked whether they recognized a pattern. Instead, they were informed that there was a pattern and asked to recall it. Explicit knowledge of the pattern was assessed using a single trial. Children were seated in front of the computer screen. The cartoon character then appeared in Position 4, and the test administrator asked the child to indicate where the character would appear next. The children indicated their responses by pointing to one of the three boxes on the screen. The test administrator encouraged the children to provide 10 responses. None of the children were able to recall the entire pattern.

Measure of vocabulary. Children's vocabulary was tested using the British Picture Vocabulary Scale, Second Edition (BPVS-II; Dunn, Dunn, Whetton, \& Pintille, 1997). The BPVS-II is a standardized test of receptive vocabulary. In this test, children are asked to match a word to one of four pictures. A receptive test of vocabulary was chosen in order to reduce the involvement of lexical recall that is considered to be supported by the basal ganglia (Ullman \& Pierpont, 2005). There are a total of 168 items on this test; however, testing is discontinued if the child makes eight or more errors in a block of 12 items. The test is standardized to a mean of 100 and an $S D$ of 15.

Measure of past tense use. Children's knowledge of the past tense was tested using Marchman et al.'s (1999) past tense task. The task consists of 52 items: 25 items assessing regular verbs and 27 items assessing irregular verbs. The items in this task are binary coded for frequency and for several phonological characteristics. In the present study, we coded for verb frequency using the Brigham Young University British National Corpus (Davies, 2004) and, using the same method as that used in Marchman et al., dichotomized the items 
using a median split of the frequency data (item frequency data are presented in Appendix A). In addition, for irregular verbs, we used the coding frame used by Marchman et al. to classify these items as having either a high or low number of regular enemies. Items with a high number of regular enemies have a larger number regular verbs that are similar in sound to the irregular past tense stem than those with a low number of regular enemies (see Appendix A). Of the total 25 regular items, 13 were high frequency. Fourteen of the 27 irregular items were high frequency and were coded as having either a high or low number of regular enemies. Of the 13 low-frequency items, seven were coded as having a high number of regular enemies.

In the past tense task, children are presented with a stimulus sentence along with a picture designed to elicit the past tense form of regular and irregular verbs. For example, on one item, which is designed to elicit the regular past tense form of the verb walk, the child is presented with a color picture of a boy walking. Then, the child is provided with the following stimulus sentence: "This boy is walking. He likes to walk every day. Yesterday he ..." , which serves to prompt the child to provide a past tense form of the verb (e.g., walked). The test sentences were prerecorded by a native speaker of Northern British English. The audio and visual stimuli were presented to children using a laptop computer, and the responses were audiotaped and coded offline.

Responses from the past tense task were coded according to the method outlined by Marchman et al. (1999). First, a response was considered as correct if the appropriate past tense form of the verb was provided. Over-regularization or zero-marking responses were considered as valid responses but coded as errors. Nonvalid responses were vowel change (e.g., brung for brought), instances in which the child's revision involved using the third person or progressive inflections, and responses that did not fit into the aforementioned categories. Only correct responses and errors were used to compute children's accuracy on the task. Nonvalid responses were not used to compute accuracy. For the purposes of this study, we obtained only those measures relevant to examining the association between declarative and procedural memory systems. The proportion of correct responses was computed for both high- and low-frequency regular and irregular verbs. This was obtained by dividing the total number of correct responses into the sum of correct and incorrect responses. In addition, for irregular verbs we computed the proportion of over-regularization errors. This was obtained by dividing the number of over-regularization errors into the total number of correct and other incorrect responses. Before presentation of the 52 test items, children were first presented with three practice items (walk, fish, and catch). In order for children to continue into the test phase, they needed to provide a valid response to these three items. All children provided a correct or valid response to these three practice items.

An independent rater coded $15 \%$ of the data for reliability. During this process, the independent rater listened to audio recordings of the children's responses and applied the previously described coding frame. There was a high degree of agreement between raters (Cohen's $\kappa=.988)$.

\section{$\overline{\text { Results }}$}

Table 2 shows descriptive statistics for the sample's scores from the measures of vocabulary, nonword repetition, and nonverbal intelligence. This table shows that all children are within $\pm 1 S D$ (i.e., the average range) on each of the tests.

\section{Preliminary Analyses From the Measures of Declarative and Procedural Memory}

In this study, the Word Pairs task was used to evaluate declarative memory. The mean score for the sample was $10.3(S D=6.3$, range $=0-25)$. Overall, the descriptive statistics indicate the absence of both ceiling and floor effects, and the distribution of scores was found to be approximately normal (see Appendix B, which shows histograms for language and memory measures).

Data from the SRT task, which was used to measure procedural memory, are now presented. Children's accuracy on the SRT task was first inspected to ensure they responded appropriately to the visual stimulus. The mean proportion of correct responses across all blocks was $.88(S D=.09 ;$ range $=.53-.98)$, which was significantly above chance level—that is, $.25, t(57)=53.122, p<.001$. Figure 2 shows mean normalized RTs reported by block. This figure shows a decline in RTs over Blocks 1-4 and an increase in Block 5. A repeated-measures analysis of variance revealed a significant effect of block on children's $\mathrm{RTs}, F(4,228)=4.546, p=.002$, partial $\eta^{2}=.073$. Planned comparisons adjusting alpha by means of the Holms procedure (Aicken \& Gensler, 1996; Holm, 1979) revealed a

Table 2. Descriptive statistics for scores from standardized tests.

\begin{tabular}{lccc}
\hline Variable & M & SD & Range \\
\hline BPVS-II & 105.8 & 10.5 & $87-124$ \\
CNRep & 105.4 & 14.8 & $84-137$ \\
RCPM & 103.6 & 13.9 & $86-141$ \\
\hline
\end{tabular}

Note. BPVS-II = British Picture Vocabulary Scale-Second Edition; CNRep = Children's Test of Nonword Repetition; RCPM = Raven's Coloured Progressive Matrices. 
Figure 2. Mean normalized reaction times (RTs), reported by block. Error bars indicate standard error.

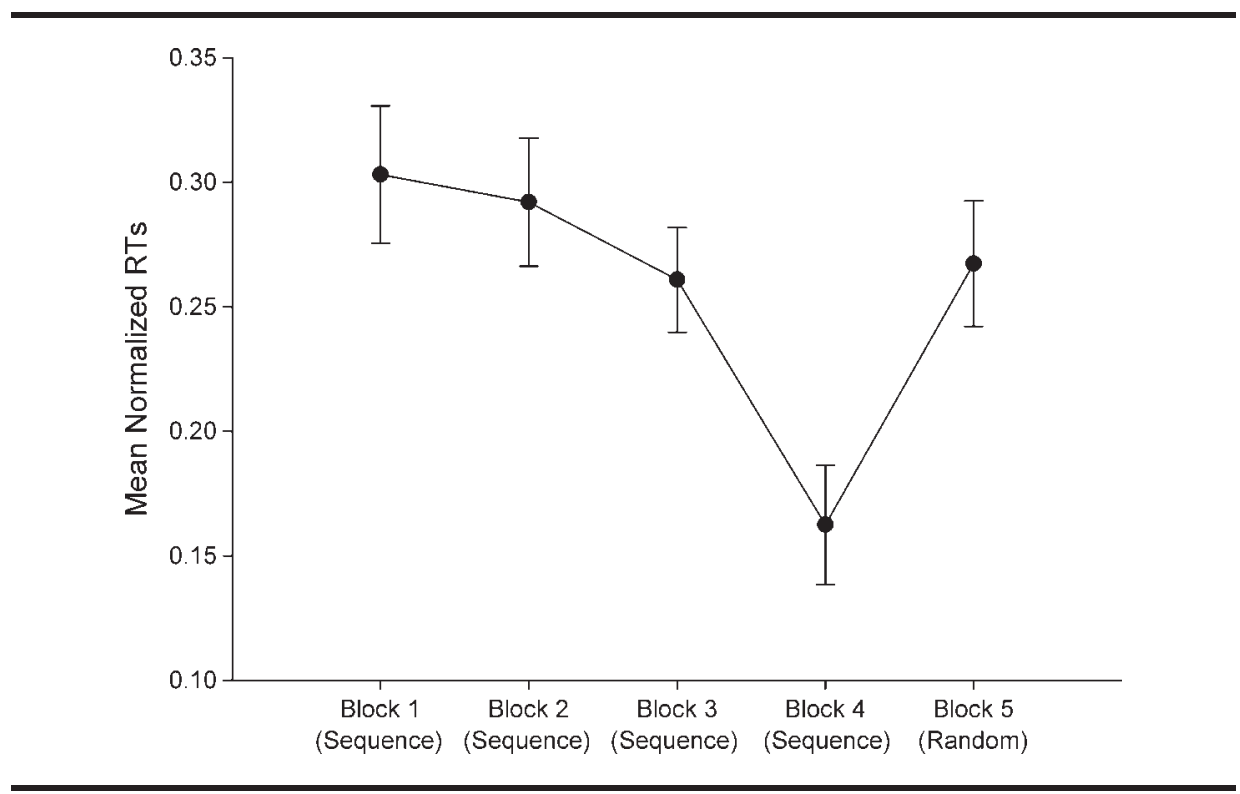

nonsignificant decrease in RTs from Block 1 to Block 2 $(p=.736)$ and Block 2 to Block $3(p=.736)$. There was a significant decrease in RTs from Block 3 to Block 4 $(p=.012)$. Finally, there was a significant increase in RTs from Block 4 to Block 5 ( $p<.001)$. Thus, across Blocks 1-4, which contained the repeating sequences, children eventually became faster at responding to the visual stimulus. However, in Block 5, which comprised random presentation of the visual stimulus, the children became slower.

\section{Results From Measures of Past Tense}

Summary statistics for the BPVS-II (which provided a measure of receptive vocabulary) and the past tense task are presented in Table 3. Raw scores from the BPVS-II and proportion of correct responses for the past tense task are reported. On the BPVS-II, as a group, floor or ceiling effects were not observed, and the distribution was found to be normal (see Appendix B).

In relation to the regular past tense items, Table 3 shows that the sample's mean score approached ceiling. The $S D$ across the different measures obtained from the past tense tasks ranged from .15 to .18 , and the proportion correct ranged from .25 to 1.0. The distribution of the scores for regular past tense items was negatively skewed (see Appendix B). In contrast, for the irregular past tense items, mean proportion of correct responses was positively skewed (see Appendix B). Inspection of the $S D$ and range of scores shows considerable variability in the performance of the sample.

A set of analyses was undertaken to examine whether children's responses on the past tense task used in this study were related to item frequency (for both regular and irregular verbs) and, for irregular items, whether over-regularizations were related to stem phonology. The goal of these analyses was to examine whether preliminary evidence could be found to support the role of declarative and procedural memory in past tense production. Specifically, Ullman (2001a, 2001c, 2004) suggested that high-frequency regular verbs may be stored and processed by the declarative memory system. Second, over-regularization errors arising from the procedural memory system may occur for low-frequency irregular items. Third, over-regularization errors that are a product of the declarative memory system are

Table 3. Summary statistics for language and memory tasks.

\begin{tabular}{|c|c|c|c|}
\hline Variable & $M$ & SD & Range \\
\hline BPVS-II: Raw score (vocabulary) & 60.1 & 11.1 & $38.0-83.0$ \\
\hline \multicolumn{4}{|l|}{ Regular past tense } \\
\hline Correct responses (all items) & 0.88 & 0.16 & $0.25-1.00$ \\
\hline High-frequency verbs & 0.94 & 0.15 & $0.25-1.00$ \\
\hline Low-frequency verbs & 0.83 & 0.18 & $0.25-1.00$ \\
\hline \multicolumn{4}{|l|}{ Irregular past tense } \\
\hline Correct responses (all items) & 0.36 & 0.18 & $0.08-0.92$ \\
\hline High-frequency verbs & 0.36 & 0.19 & $0.06-0.92$ \\
\hline Low-frequency verbs & 0.36 & 0.19 & $0.05-0.92$ \\
\hline Over-regularization errors (all items) & 0.47 & 0.22 & $0.08-0.90$ \\
\hline High-frequency verbs & 0.48 & 0.24 & $0.06-0.89$ \\
\hline High regular enemies & 0.45 & 0.28 & $0.00-1.00$ \\
\hline Low regular enemies & 0.41 & 0.25 & $0.00-1.00$ \\
\hline Low-frequency verbs & 0.47 & 0.21 & $0.08-0.90$ \\
\hline High regular enemies & 0.53 & 0.26 & $0.00-1.00$ \\
\hline Low regular enemies & 0.45 & 0.20 & $0.17-0.83$ \\
\hline
\end{tabular}


proposed to occur for those irregular verbs that are similar in sound to regular verbs (i.e., have a high number of regular enemies). In order to test these claims it was considered necessary to examine whether the coding of items as either high or low frequency or as having either a high or low number of regular enemies had an observable impact on children's responses.

Differences in high- and low-frequency regular past tense items were examined using a paired samples $t$ test. Because the distribution of regular past tense scores was negatively skewed (see Appendix B), $p$ values were estimated using a bootstrapping procedure. Bootstrapping procedures involve repeatedly taking samples with replacement from the sample data to estimate population parameters (Efron \& Tibshirani, 1993). Research has demonstrated this approach to be effective in estimating population parameters for non-normal data (Field, 2009). The results from the paired samples $t$ test revealed that children scored significantly higher on high-frequency regular past tense items compared with low-frequency items, $t(57)=7.086, p<.001$, partial $\eta^{2}=.468$. The observed effect size for this analysis was found to be large (Cohen, 1988). ${ }^{1}$

For irregular verbs, there was no significant difference in accuracy between high- and low-frequency items, $t(57)=.076, p=.939$, partial $\eta^{2}<.001$, and the observed effect size was small. However, frequency and phonological effects were observed for over-regularization errors. The effects of frequency and stem phonology were examined using a 2 (Frequency: High, Low) $\times 2$ (Neighborhood Density: High Regular Enemies, Low Regular Enemies) repeated-measures analysis of variance. The dependent variable in this analysis was the proportion of overregularization errors. This analysis revealed a significant main effect for frequency, $F(1,57)=8.146, p=.006$, partial $\eta^{2}=.125$, and stem phonology, $F(1,57)=10.646, p=.002$, partial $\eta^{2}=.157$. That is, children made significantly more over-regularization errors for low-frequency verbs and for irregular verbs with a high number of regular enemies.

\section{Relationship Between Memory and Language Measures}

The next set of analyses examined associations between the measures of memory and language. First, bivariate correlations were computed between the measures of declarative memory (measured with the Word Pairs task), receptive vocabulary (measured with the BPVS-II), and past tense use. Bivariate correlations were also computed using a measure of procedural memory

\footnotetext{
${ }^{1}$ For partial $\eta^{2}, .138$ is considered large, .059 is considered medium, and .010 is considered small.
}

from the SRT task, receptive vocabulary, and past tense. A measure of procedural memory was obtained by subtracting RTs in Block 4 from those of Block 5. This approach has been previously used to quantify procedural learning on this task (Siegert et al., 2006; Thomas et al., 2004). According to this index, values greater than zero indicate that information about the sequence is retained. In this sample, the mean normalized RT between these two blocks was .10, with an $S D$ of .20 (range $=-.31-.58$ ). The distribution of this variable was normal (see Appendix B). Power analysis revealed that for the bivariate correlations, there was a $79.8 \%$ chance of detecting significant effect when the coefficient was .360. It should be noted that it is generally recommended that statistical tests have an $80 \%$ chance of detecting a significant effect (Field, 2009).

Second, partial correlations were computed to examine the association between memory and language while controlling for extraneous variables. Specifically, to examine the association among declarative memory, vocabulary, and past tense, it was necessary to control for the influence of procedural memory. In these analyses, the covariates used were the measure of procedural memory described above and RCPM scores. For these partial correlations, power analysis revealed there was a $79.2 \%$ chance of detecting a correlation coefficient of .370.

To examine the association between procedural memory and the language measures, it was necessary to control for the influence of declarative memory. Thus, scores from the Word Pairs task were used as a covariate. Additional covariates included in the analyses were vocabulary (measured using the BPVS-II) and phonological short-term memory (measured using the CNRep), given their proposed involvement in declarative memory (Foerde et al., 2006; Ullman \& Pierpont, 2005). The final covariate used was RCPM scores. For this set of partial correlations, there was an $80 \%$ chance of detecting a correlation of .380.

Table 4 shows bivariate and partial correlations between the memory and language measures. This table shows that the only statistically significant correlation observed was between the measure of declarative memory (measured using the Word Pairs task) and vocabulary (measured using the BPVS-II). This correlation was observed after controlling for general intelligence (measured using the RCPM) and procedural memory. The measure of declarative memory was not found to be correlated with any of the variables derived from the past tense task. Relevant to evaluating the hypotheses in this study were the nonsignificant correlations observed between declarative memory and the proportion of correct responses for irregular past tense use, over-regularizations for high-frequency irregular items, and those with a high number of regular enemies. According to Cohen's (1988) taxonomy, the magnitude of 
Table 4. Bivariate and partial correlations between memory and language variables.

\begin{tabular}{|c|c|c|c|c|}
\hline \multirow[b]{3}{*}{ Language measures } & \multirow{2}{*}{\multicolumn{2}{|c|}{ Declarative memory ${ }^{a}$}} & \multicolumn{2}{|r|}{ Procedural memory ${ }^{b}$} \\
\hline & & & & \multirow{2}{*}{$\begin{array}{c}\text { Controlling for RCPM, } \\
\text { declarative memory, vocabulary, } \\
\text { and phonological working memory }\end{array}$} \\
\hline & No covariates & $\begin{array}{l}\text { Controlling for RCPM } \\
\text { and procedural memory }\end{array}$ & No covariates & \\
\hline Vocabulary (BPVS-II) & $.436^{*}$ & $.438^{*}$ & & \\
\hline \multicolumn{5}{|l|}{ Regular past tense } \\
\hline Correct responses (all items) & .071 & .053 & .115 & .095 \\
\hline High-frequency verbs & .009 & -.020 & .163 & .174 \\
\hline Low-frequency verbs & .112 & .103 & .072 & .032 \\
\hline \multicolumn{5}{|l|}{ Irregular past tense } \\
\hline Correct responses (all items) & .177 & .182 & -.007 & -.089 \\
\hline High-frequency verbs & .208 & .213 & -.009 & -.094 \\
\hline Low-frequency verbs & .147 & .151 & -.005 & -.080 \\
\hline Over-regularization errors (all items) & -.088 & -.054 & .029 & .061 \\
\hline High-frequency verbs & -.088 & -.062 & .049 & .077 \\
\hline High regular enemies & -.018 & -.008 & .170 & .171 \\
\hline Low regular enemies & -.015 & .032 & -.020 & -.013 \\
\hline Low-frequency verbs (all items) & -.086 & -.046 & .011 & .058 \\
\hline High regular enemies & -.239 & -.217 & -.005 & .071 \\
\hline Low regular enemies & -.057 & .112 & -.016 & -.011 \\
\hline
\end{tabular}

all these coefficients can be considered small. ${ }^{2}$ The measure of procedural memory was also not found to be significantly correlated with any of the past tense measures, including the proportion of correct responses for regular verbs and over-regularization errors. The magnitude of these correlation coefficients can be considered small.

\section{$\overline{\text { Discussion }}$}

In this study, we examined associations among measures of procedural/declarative memory, past tense, and vocabulary. To our knowledge, this is one of the first reports to evaluate whether the types of associations between memory and language predicted by the D \& P model (Ullman, 2001a, 2001c, 2004) can be observed in typically developing 5 -year-old children. Overall, there was limited evidence supporting the D \& P model. Consistent with the model, the correlation between declarative memory and vocabulary was found to be statistically significant after controlling for intellectual development and procedural memory. However, inconsistent with the model, declarative memory was not found to be correlated with irregular past tense verbs, high-frequency regular verbs, or over-regularization errors. Data from the procedural memory task also failed to support the hypothesis that this memory system

$\overline{{ }^{2} \text { For correlation coefficients }}, .371$ is considered large, .243 is considered medium, and .100 is considered small. would be correlated with regular past tense use. Collectively, the results of the study question the involvement of declarative and procedural systems in typically developing 5-year-old children's production of regular and irregular past tense. When interpreting these results, it is first important to note the correlational nature of the design of the study. Thus, the absence or presence of correlations may be explained with reference to one or more nonmeasured variables. Thus, additional research is required to examine whether the results reported in this study can be replicated.

One prediction of the D \& P model supported by the results was the significant correlations observed between the measure of declarative memory and vocabulary. It is important to note that this correlation was still found to be significant after controlling for individual differences in procedural memory and general cognitive development (as indexed by the RCPM). Thus, the associations observed between declarative memory and this component of language appears robust. It is likely that the relationship between declarative memory and vocabulary is bidirectional (Gathercole, Hitch, Service, $\&$ Martin, 1997). Specifically, children who are better able to encode and retrieve verbal information may be more efficient at learning new words. Alternatively, children with larger vocabularies presumably have larger semantic and phonological associative networks that may be more easily able to accommodate newer words. Even assuming a bidirectional relationship, this result supports 
Ullman's (2001a, 2001c, 2004) claims that the capacity of the declarative memory system to encode and retrieve arbitrarily related form and meaning associations may be important for word learning.

As previously noted, the predictions concerning past tense use derived from the $\mathrm{D} \& \mathrm{P}$ model were not supported. In the first instance, it is notable that the preliminary analyses undertaken with the past tense data indicated the presence of frequency and stem phonology effects in past tense use. This result is consistent with the findings by Marchman et al. (1999) using the same task. Based on the arguments previously forwarded by Ullman (Hartshorne \& Ullman, 2006; Ullman, 2001a, 2001c, 2004), the children's performance on the highfrequency past tense items and irregular verbs with a high number of regular enemies, in principal, should be associated with declarative memory. However, despite the presence of these effects, the measure of declarative memory did not correlate with irregular- or high-frequency regular verbs.

In considering the nonsignificant results, issues relating to statistical power and task sensitivity need to be considered. First, it does not seem that the null results reflect problems with statistical power. With a sample size of 58 and four covariates, there was an $80.3 \%$ probability of detecting a statistically significant correlation coefficient as low as .365. The magnitude of the partial correlations computed between the memory systems and past tense presented in Table 4 ranged from -.217 to .213. According to Cohen's (1988) taxonomy, these values fall between small to medium in magnitude. There would have been an $80 \%$ chance of returning a significant correlation if sample sizes of 164 and 170 , respectively, had been used. ${ }^{3}$ However, this post hoc power analysis raises questions about whether observing significant correlations of this magnitude are empirically meaningful. Second, it could be argued that the measures of declarative memory (as measured by the Word Pairs Task) and procedural memory (as measured by subtracting the difference between Block 4 and Block 5) were not sensitive to individual differences. However, such an argument would be inconsistent with the significant correlations observed between the Word Pairs Task and BPVS-II. Also, on the SRT task, significant differences were observed between sequenced and random blocks, a result that is consistent with other research examining procedural memory in nonclinical populations (e.g., Thomas \& Nelson, 2001).

It could also be argued that there was insufficient variability in the measure of regular and irregular past tense because in both cases the distributions were skewed. If this were the case, we might expect that performance on the regular past tense task would

\footnotetext{
${ }^{3}$ Assuming an alpha level of .05 and a two-tailed test.
}

not correlate with any of the measures. However, as a post hoc analysis, we find that BPVS-II scores are significantly correlated with the proportion of correct responses for regular past tense $(r=.337, p=.011)$ and irregular past tense use ( $r=.357, p=.007)$, even after controlling for RCPM scores. Thus, there is evidence that there is sufficient variability in the past tense task scores such that performance correlates with vocabulary. Thus, an outstanding issue to be addressed is how the $\mathrm{D} \&$ P model might account for the nonsignificant association between past tense and measures of declarative and procedural memory.

The age of the participants might be an important variable relevant to understanding the nonsignificant results. Children participating in this study were approximately 5 years old. An assumption made in this study was that declarative and procedural memory systems equally support past tense use at this age. An alternative position could be that because declarative and procedural memory systems are functionally mature at different points in development (Reber, 1993), their respective influence on past tense use might not be constant as children learn language. For instance, the children in this study were already approaching ceiling on the items assessing regular past tense. This finding has been observed in other studies undertaken with English-speaking children (e.g., Rice \& Wexler, 1998). Given this, it could be that the influence of procedural memory on grammar and syntax is prominent earlier in language development. In contrast, the children performed quite poorly on the irregular past tense items. Across all irregular items, the mean proportion of correct responses was .36. This result is also consistent with other research undertaken with typically developing children approximately 5 years old (Rice et al., 2000). Because the acquisition of irregular verbs occurs when children are older than 5 , the declarative memory system might play a more important role in this aspect of language later in development.

Measurement issues relating to the assessment of procedural memory should also be taken into account. In this study, procedural memory was assessed using an SRT task in which children implicitly learned a visuospatial sequence. However, yet to be investigated is whether paradigms that assess the implicit learning of information in the verbal domain (e.g., Saffran, Aslin, $\&$ Newport, 1996) correlate with past tense use. Should an association be observed in the verbal, but not visual, domain, a challenge for the $\mathrm{D} \& \mathrm{P}$ model would be to account for such a discrepancy when both task assess the same brain structure that is hypothesized to underlie grammar.

It is also acknowledged that the results of this study do not discount other models of grammar that assume varying degrees of independence between the acquisition and use of regular and irregular verbs and nouns. 
For example, like the D \& P model, Pinker's (1999) words and rules model also holds that regular verbs and nouns are derived through deploying combinatorial operations. Also, irregular verbs and nouns along with lexical items are stored as whole units. However, an important difference between these two approaches is that only the $\mathrm{D} \& \mathrm{P}$ model is committed to aligning the regular verbs and nouns to the procedural memory system and irregular forms and lexical items to the declarative memory system. Thus, within the literature of dual route approaches to lexical knowledge and grammar, the results of this study question whether declarative and procedural memory systems support different components of language. However, the results are certainly not dismissive of other dual route models that are noncommittal about the different memory systems implicated in language.

\section{Conclusions}

In the present study, we investigated the associations among memory, past tense, and vocabulary in typically developing 5-year-old children. Overall, the data did not indicate that measures of declarative or procedural memory were linearly related to children's past tense use. However, after controlling for extraneous variables, declarative memory was found to be significantly correlated with vocabulary. Overall, these results question whether declarative and procedural memory support past tense use in typically developing 5 -year-old children as predicted by the D \& P model. However, whether this holds across development is yet to be tested.

\section{Acknowledgments}

This research was supported by British Academy Grant SG-42144 (awarded to both authors) and a Charles La Trobe Research Fellowship (awarded to the second author). We thank Rachael King and Sarah Davis for help in testing the children, and we thank the schools and children who took part in this study.

\section{References}

Aicken, M., \& Gensler, H. (1996). Adjusting for multiple testing when reporting research results: The Bonferroni vs Holm methods. Public Health Briefs, 86, 726-728.

Anderson, V., Elisabeth, N., Hendy, J., \& Wrennall, J. (2001). Developmental neuropsychology: A clinical approach. Hove, United Kingdom: Psychology Press.

Anderson, V. A., \& Lajoie, G. (1996). Development of memory and learning skills in school-aged children: A neuropsychological perspective. Applied Neuropsychology, 3, 128-139.

Baddeley, A. (2003). Working memory: Looking back and looking forward. Nature Reviews Neuroscience, 4, 829-839.

Bates, E., \& Goodman, J. C. (1997). On the inseperability of grammar and the lexicon: Evidence from acquisition, aphasia, and real-time processing. Language and Cognitive Processes, 12, 507-584.

Bishop, D. V., Adams, C. V., \& Norbury, C. F. (2006). Distinct genetic influences on grammar and phonological short-term memory deficits: Evidence from 6-year-old twins. Genes, Brain and Behavior, 5, 158-169.

Bishop, D. V. M., North, T., \& Donlan, C. (1996). Nonword repetition as a behavioural marker for inherited language impairment: Evidence from a twin study. Journal of Child Psychology and Psychiatry, 37, 391-403.

Bybee, J., \& Slobin, D. I. (1982). Rules and schemas in the development and use of the English past tense. Language, $58,265-289$

Chalmers, S. L., \& Frederick, C. O. (1955). The relationship between Raven's Coloured Progressive Matrices and two tests of general intelligence. Journal of Clinical Psychology, 11, 84-85.

Cohen, J. (1988). Statistical power analysis for the behavioral sciences (2nd ed.). Hillsdale, NJ: Erlbaum.

Cohen, J. (1997). Children's Memory Scales. London, United Kingdom: The Psychological Corporation.

Conti-Ramsden, G., Botting, N., \& Faragher, B. (2001). Psycholinguistic markers for specific language impairment (SLI). Journal of Child Psychology and Psychiatry and Allied Disciplines, 42, 741-748.

Cowan, N. (1997). Attention and memory: An integrated framework. New York, NY: Oxford University Press.

Davies, M. (2004). BYU-BNC: The British National Corpus. Retrieved from http://corpus.byu.edu/bnc.

Dunn, L. M., Dunn, L. M., Whetton, C., \& Pintille, D. (1997). British Picture Vocabulary Scale (2nd ed.). Berkshire, United Kingdom: NFER-Nelson.

Efron, B., \& Tibshirani, R. (1993). An introduction to the bootstrap (Vol. 57). London, United Kingdom: Chapman \& Hall/CRC.

Eichenbaum, H., \& Bunsey, M. (1995). On the binding of associations in memory: Clues from studies on the role of the hippocampal region in paired-associate learning. Current Directions in Psychological Science, 4, 19-23.

Engle, R. W., Cantor, J., \& Carullo, J. J. (1992). Individual differences in working memory and comprehension: A test of four hypotheses. Journal of Experimental Psychology: Learning, Memory, and Cognition, 18, 972-992.

Field, A. P. (2009). Discovering statistics using SPSS (and sex and drugs and rock ' $n$ ' roll). London, United Kingdom: Sage.

Foerde, K., Knowlton, B. J., Poldrack, R. A., \& Smith, E. E. (2006). Modulation of competing memory systems by distraction. Proceedings of the National Academy of Sciences of the United States of America, 103, 11778-11783.

Gathercole, S. E. (1995). Is nonword repetition a test of phonological memory or long-term knowledge? It all depends on the nonwords. Memory \& Cognition, 23, 83-94.

Gathercole, S. E., \& Baddelely, A. D. (1996). Children's Test of Nonword Repetition. London, United Kingdom: Harcourt Assessment.

Gathercole, S. E., Hitch, G. J., Service, E., \& Martin, A. J. (1997). Short-term memory and new word learning in children. Developmental Psychology, 33, 966-979. 
Gathercole, S. E., Pickering, S. J., Ambridge, B., \& Wearing, H. (2004). The structure of working memory from 4 to 15 years of age. Developmental Psychology, 40, 177-190.

Giedd, J. N., Blumenthal, J., Jeffries, N. O., Castellanos, F. X., Liu, H., Zijdenbos, A., ... Rapoport, J. L. (1999). Brain development during childhood and adolescence: A longitudinal MRI study. Nature Neuroscience, 2, 861-862.

Graf Estes, K., Evans, J. L., \& Else-Quest, N. M. (2007). Differences in the nonword repetition performance of children with and without specific language impairment: A meta-analysis. Journal of Speech, Language, and Hearing Research, 50, 177-195.

Hartshorne, J. K., \& Ullman, M. T. (2006). Why girls say 'holded' more than boys. Developmental Science, 9, 21-32.

Hikosaka, O., Nakamura, K., Sakai, K., \& Nakahara, H. (2002). Central mechanisms of motor skill learning. Current Opinion in Neurobiology, 12, 217-222.

Holm, S. (1979). A simple sequentially rejective multiple testing procedure. Scandinavian Journal of Statistics, 6, 65-70.

Iaria, G., Petrides, M., Dagher, A., Pike, B., \& Bohbot, V.D. (2003). Cognitive strategies dependent on the hippocampus and caudate nucleus in human navigation: Variability and change with practice. Journal of Neuroscience, 23, 5945-5952.

Jambaqué, I., Dellatolas, G., Fohlen, M., Bulteau, C., Watier, L., Dorfmuller, G., ... Delalande, O. (2007). Memory functions following surgery for temporal lobe epilepsy in children. Neuropsychologia, 12, 2850-2862.

Joanisse, M. F., \& Seidenberg, M. S. (1998). Specific language impairment: A deficit in grammar or processing? Trends in Cognitive Sciences, 2, 240-247.

Knopman, D., \& Nissen, M. J. (1991). Procedural learning is impaired in Huntington's disease: Evidence from the serial reaction time task. Neuropsychologia, 29, 245-254.

Knowlton, B. J., Mangels, J. A., \& Squire, L. R. (1996, September 6). A neostriatal habit learning system in humans. Science, 273, 1399-1402.

Kramer, J. H., Delis, D. C., Kaplan, E., O’Donnell, L., \& Prifitera, A. (1997). Developmental sex differences in verbal learning. Neuropsychology, 11, 577-584.

Lum, J. A. G., Gelgec, C., \& Conti-Ramsden, G. (2010), Procedural and declarative memory in children with and without specific language impairment. International Journal of Language \& Communication Disorders, 45, 96-107.

Lum, J. A. G., Kidd, E., Davis, S., \& Conti-Ramsden, G. (2010). Longitudinal study of declarative and procedural memory in primary school-aged children. Australian Journal of Psychology, 62, 139-148.

Marchman, V. A., Wulfeck, B., \& Ellis Weismer, S. (1999). Morphological productivity in children with normal language and SLI: A study of the English past tense. Journal of Speech, Language, and Hearing Research, 42, 206-219.

Marcus, G. F., Pinker, S., Ullman, M., Hollander, M., Rosen, T. J., \& Xu, F. (1992). Overregularization in language acquisition. Monographs of the Society for Reserach in Child Development, 57, 1-178.

Mayes, A., Montaldi, D., \& Migo, E. (2007). Associative memory and the medial temporal lobes. Trends in Cognitive Sciences, 11, 126-135.
Meulemans, T., Van der Linden, M., \& Perruchet, $\mathbf{P}$. (1998). Implicit sequence learning in children. Journal of Experimental Child Psychology, 69, 199-221.

Nissen, M. J., \& Bullemer, P. (1987). Attentional requirements of learning: Evidence from performance measures. Cognitive Psychology, 19, 1-32.

Packard, M. G., \& Knowlton, B. J. (2002). Learning and memory functions of the basal ganglia. Annual Review of Neuroscience, 25, 563-593.

Pinker, S. (1991, August 2). Rules of language. Science, 253, $530-535$.

Pinker, S. (1999). Words and rules: The ingredients of language. New York, NY: Basic Books.

Pinker, S., \& Ullman, M. T. (2002). The past and future of past tense. Trends in Cognitive Sciences, 6, 463-465.

Poldrack, R. A., Clark, J., Pare-Blagoev, E. J., Shohamy, D., Moyano, J. C., Myers, C., \& Gluck, M. (2001, November 29). Interactive memory systems in the human brain. Nature, 414, 546-550.

Poldrack, R. A., \& Gabrieli, J. D. E. (2001). Characterizing the neural mechanisms of skill learning and repetition priming. Brain, 124, 67-82.

Poldrack, R. A., \& Packard, M. G. (2003). Competition among multiple memory systems: Converging evidence from animal and human brain studies. Neuropsychologia, 41, 245-251.

Poldrack, R. A., \& Rodriguez, P. (2004). How do memory systems interact? Evidence from human classification learning. Neurobiology of Learning and Memory, 82, 324-332.

Raven, J. (1998). Raven's Coloured Progressive Matrices and Vocabulary Scales. London, United Kingdom: Psychological Corporation

Reber, A. S. (1993). Implicit learning and tacit knowledge: An essay on the cognitive unconscious. New York, NY: Oxford University Press.

Rice, M. (2000). Grammatical symptoms of specific language impairment. In D. V. M. Bishop \& L. B. Leonard (Eds.), Speech and language impairments in children: Causes, characteristics, intervention and outcome (pp. 17-34). East Sussex, United Kingdom: Psychology Press.

Rice, M. L., \& Wexler, K. (1996). Toward tense as a clinical marker of specific language impairment in English-speaking children. Journal of Speech and Hearing Research, 39, 1239-1257.

Rice, M. L., \& Wexler, K. (1998). Tense over time: The longitudinal course of tense acquisition in children with specific language impairment. Journal of Speech, Language, and Hearing Research, 41, 1412-1431.

Rice, M. L., Wexler, K., \& Cleave, P. (1995). Specific language impairment as a period of extended optional infinitive. Journal of Speech and Hearing Research, 38, 850-863.

Rice, M. L., Wexler, R., Marquis, J., \& Hershberger, S. (2000). Acquisition of irregular past tense by children with specific language impairment. Journal of Speech, Language, and Hearing Research, 43, 1126-1145.

Robertson, E., Tormos, J., Maeda, F., \& Pascual-Leone, A. (2001). The role of the dorsolateral prefrontal cortex during sequence learning is specific for spatial information. Cerebral Cortex, 11, 628-635.

Saffran, J. R., Aslin, R. N., \& Newport, E. L. (1996, December 13). Statistical learning by 8-month old infants. Science, 274, 1926-1928. 
Siegert, R. J., Taylor, K. D., Weatherall, M., \& Abernethy, D. A. (2006). Is implicit sequence learning impaired in Parkinson's disease? A meta-analysis. Neuropsychology, 20, 490-495.

Squire, L. R., Stark, C. E. L., \& Clark, R. (2004). The medial temporal lobe. Annual Review of Neuroscience, 27, 279-306.

Thomas, K. M., Hunt, R. H., Vizueta, N., Sommer, T., Durston, S., \& Yang, Y. (2004). Evidence of developmental differences in implicit sequence learning: An fMRI study of children and adults. Journal of Cognitive Neuroscience, 16, 1339-1351.

Thomas, K. M., \& Nelson, C. A. (2001). Serial reaction time learning in preschool- and school-age children. Journal of Experimental Child Psychology, 79, 364-387.

Tomblin, J. B., Mainela-Arnold, E., \& Zhang, X. (2007). Procedural learning in adolescents with and without specific language impairment. Language Learning and Development, 3, 269-293.

Ullman, M. T. (2001a). The declarative/procedural model of lexicon and grammar. Journal of Psycholinguistic Research, $30,37-69$.

Ullman, M. T. (2001b). The neural basis of lexicon and grammar in first and second language: The declarative/procedural model. Bilingualism: Language and Cognition, 4, 105-122.

Ullman, M. T. (2001c). A neurocognitive perspective on language: The declarative/procedural model. Nature Reviews Neuroscience, 2, 717-726.

Ullman, M. T. (2004). Contributions of memory circuits to language: The declarative/procedural model. Cognition, $92,231-270$.

Ullman, M. T., Corkin, S., Coppola, M., Hickok, G., Growdon, J. H., Koroshetz, W. J., \& Pinker, S. (1997). A neural dissociation within language: Evidence that the mental dictionary is part of declarative memory, and that grammatical rules are processed by the procedural system. Journal of Cognitive Neuroscience, 9, 266-276.
Ullman, M. T., Pancheva, R., Love, T., Yee, E., Swinney, D., \& Hickok, G. (2005). Neural correlates of lexicon and grammar: Evidence from the production, reading, and judgment of inflection in aphasia. Brain and Language, 93, $185-238$.

Ullman, M. T., \& Pierpont, E. L. (2005). Specific language impairment is not specific to language: The procedural deficit hypothesis. Cortex, 41, 399-433.

van der Lely, H. K., \& Ullman, M. T. (2001). Past tense morphology in specifically language impaired and normally developing children. Language and Cognitive Processes, 16, 177-217.

Walenski, M., Motofsky, S. H., \& Ullman, M. T. (2007). Speeded processing of grammar and tool knowledge in Tourette's syndrome. Neuropsychologia, 45, 2447-2460.

Walenski, M., Sosta, K., Cappa, S., \& Ullman, M. T. (2009). Deficits on irregular verbal morphology in Italian-speaking Alzheimer's disease patients: Evidence from present tense and past participle production. Neuropsychologia, 47, $1245-1255$.

Walenski, M., Weickert, T. W., Maloof, C. J., \& Ullman, M. T. (2010). Grammatical processing in schizophrenia: Evidence from morphology. Neuropsychologia, 48, 262-269.

Weismer, S. E., Tomblin, J. B., Zhang, X., Buckwalter, P., Chynoweth, J. G., \& Jones, M. (2000). Nonword repetition performance in school-age children with and without language impairment. Journal of Speech, Language, and Hearing Research, 43, 865.

Wood, A. G., Saling, M. M., O'Shea, M. F., Berkovic, S. F., \& Jackson, G. D. (2000). Components of verbal learning and hippocampal damage assessed by $\mathrm{T} 2$ relaxometry. Journal of the International Neuropsychology Society, 6, 529-538. 
Appendix A (p. 1 of 2). Past tense item frequencies.

\begin{tabular}{|c|c|c|c|}
\hline Past tense item & Frequency type & Frequency count ${ }^{a}$ & Regular enemies $^{b}$ \\
\hline \multicolumn{4}{|l|}{ Regular past tense item } \\
\hline Tried & High & 112.9 & \\
\hline Needed & High & 80.3 & \\
\hline Played & High & 71.7 & \\
\hline Picked & High & 69.0 & \\
\hline Talked & High & 68.5 & \\
\hline Smiled & High & 33.0 & \\
\hline Watched & High & 32.3 & \\
\hline Loved & High & 22.3 & \\
\hline Jumped & High & 15.3 & \\
\hline Spelled/spelt & High & 13.9 & \\
\hline Baked & High & 7.9 & \\
\hline Tasted & High & 3.7 & \\
\hline Matched & High & 3.1 & \\
\hline Kissed & Low & 3.0 & \\
\hline Danced & Low & 1.8 & \\
\hline Mended & Low & 1.8 & \\
\hline Leaned/leant & Low & 1.2 & \\
\hline Melted & Low & 1.1 & \\
\hline Flowed & Low & 0.9 & \\
\hline Peeled & Low & 0.8 & \\
\hline Spilled & Low & 0.6 & \\
\hline Hugged & Low & 0.5 & \\
\hline Drummed & Low & 0.4 & \\
\hline Raked & Low & 0.4 & \\
\hline Skated & Low & 0.2 & \\
\hline
\end{tabular}


Appendix A (p. 2 of 2). Past tense item frequencies.

\begin{tabular}{|c|c|c|c|}
\hline Past tense item & Frequency type & Frequency count $^{a}$ & Regular enemies ${ }^{\mathrm{b}}$ \\
\hline \multicolumn{4}{|c|}{ Irregular past tense item } \\
\hline Went & High & 953.9 & High \\
\hline Bit & High & 667.1 & High \\
\hline Told & High & 376.6 & High \\
\hline Sent & High & 114.2 & High \\
\hline Stuck & High & 71.2 & High \\
\hline Fell & High & 47.3 & High \\
\hline Took & High & 297.8 & Low \\
\hline Brought & High & 148.6 & Low \\
\hline Felt & High & 112.9 & Low \\
\hline Cut & High & 101.7 & Low \\
\hline Hit & High & 86.8 & Low \\
\hline Built & High & 85.7 & Low \\
\hline Sat & High & 80.3 & Low \\
\hline Held & High & 58.3 & Low \\
\hline Drove & Low & 17.9 & High \\
\hline Threw & Low & 15.6 & High \\
\hline Ate & Low & 15.6 & High \\
\hline Fed & Low & 14.1 & High \\
\hline Shed & Low & 10.9 & High \\
\hline Flew & Low & 9.4 & High \\
\hline Drank & Low & 4.3 & High \\
\hline Ran & Low & 41.9 & Low \\
\hline Broke & Low & 37.3 & Low \\
\hline Hurt & Low & 21.9 & Low \\
\hline Drew & Low & 10.6 & Low \\
\hline Sang & Low & 6.6 & Low \\
\hline Rode & Low & 0.3 & Low \\
\hline
\end{tabular}

Note. Blank cells indicate "not applicable."

${ }^{a}$ Expressed as tokens per million spoken words. ' ${ }^{\circ}$ rom Marchman et al. (1999).

Downloaded From: http://jslhr.pubs.asha.org/ by a Max Planck Institut User on 11/05/2017 Terms of Use: http://pubs.asha.org/ss/rights_and_permissions.aspx 
Appendix B. Distribution of scores for Word Pairs (declarative memory), SRT (procedural memory), BPVS-II (receptive vocabulary), and past tense tasks.
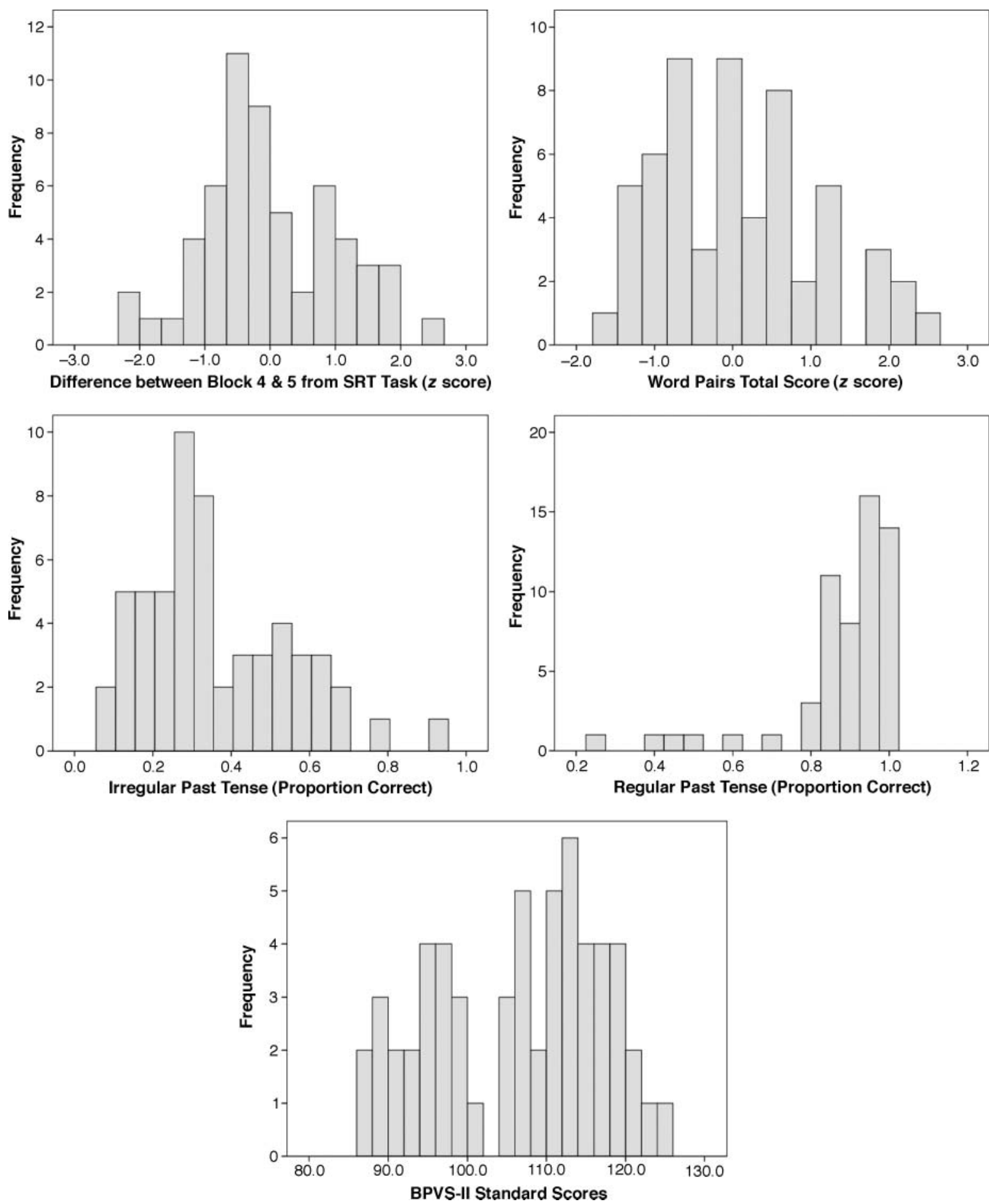